\title{
Intra-abdominal Actinomycosis Imitating Sigmoid Colon Cancer: A Case Report
}

\author{
Ramazan Sari ${ }^{1, *}$, Murat Kuş ${ }^{1}$, Fazilet Kayaselçuk ${ }^{2}$
}

1. Department of General Surgery, Baskent University, Adana, Turkey

2. Department of Pathology, Baskent University, Adana, Turkey

\section{* Corresponding Author:}

Ramazan Sari, MD

Orhantepe mah. Guzelbahce sk. No: 10/A Kartal, Istanbul, Turkey

Tel: +905056294662

Fax: + 902163520083

Email: sariramazan71@gmail.com

Received: 11 Dec. 2019

Accepted: 28 Feb 2020

\section{ABSTRACT}

Actinomycosis is a rare bacterial disease characterized by chronic or subacute suppurative inflammation. Abdominal actinomycosis is rarer and accounts for approximately $20 \%$ of all actinomycosis cases. In the literature, patients who underwent surgery for actinomycosis mimicking malignant tumors have been reported. Most of these patients had mucosal trauma and inflammation signs. It is often difficult to diagnose abdominal actinomycosis preoperatively and often impossible to distinguish it from a malignant tumor. We present a case that preoperatively was diagnosed as a malignant tumor but pathological evaluation reported to be diverticulitis and actinomycosis. This case can increase the awareness about this disease, which is possible to be treated non-surgically.

\section{KEYWORDS:}

Actinomycosis, Abdominal mass, Sigmoid colon

\section{Please cite this paper as:}

Sari R, Kuş M, Kayaselçuk F. Intra-abdominal Actinomycosis Imitating Sigmoid Colon Cancer: A Case Report. Middle East J Dig Dis 2020;12:126-129. doi: 10.34172/ mejdd.2020.173.

\section{INTRODUCTION}

Actinomycosis is an infectious disease that is rarely seen and usually presents with chronic or subacute suppurative inflammation. ${ }^{1}$ In humans, the most common cause of this disease is Actinomyces Israelii, which took the name of the first person who identified it during human autopsies. ${ }^{2}$ This gram-positive anaerobic bacteria is found in the oral, gastrointestinal, and urogenital mucosa and can sometimes cause opportunistic infections. Cervicofacial actinomycosis is the most common (60\%) and abdominal actinomycosis is rarer, which accounts for approximately $20 \%$ of all cases. ${ }^{3}$ It usually occurs after mucosal damage caused by appendicitis, diverticulitis, and intrauterine device (IUD). It is difficult to diagnose and may mimic a malignant tumor clinically and radiologically. ${ }^{4}$ For this reason, the surgical decision is usually made for the treatment and the definite diagnosis is confirmed histopathologically. Herein, we present a case of abdominal actinomycosis that was detected after resection due to suspicion of malignancy in a patient without a prominent clinical presentation. Our aim is to find out if it is possible to recognize this rare phenomenon earlier and question if surgical intervention is at all necessary. 


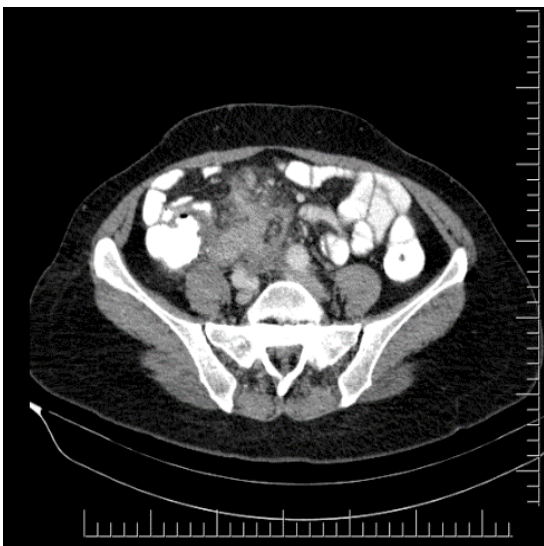

Fig.1: Computed tomography

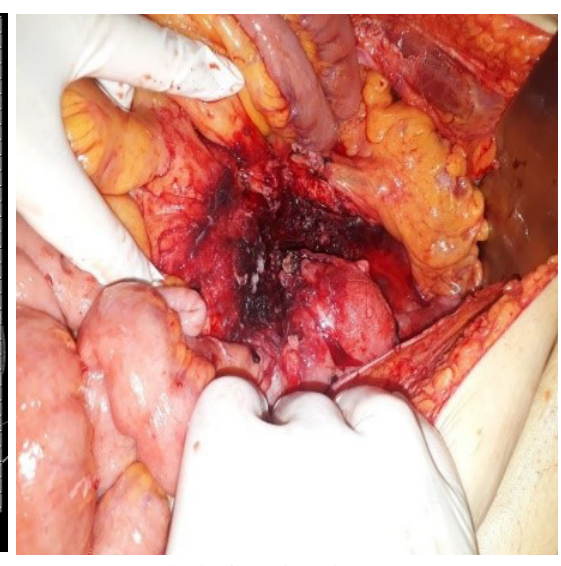

Fig.2: Surgical image

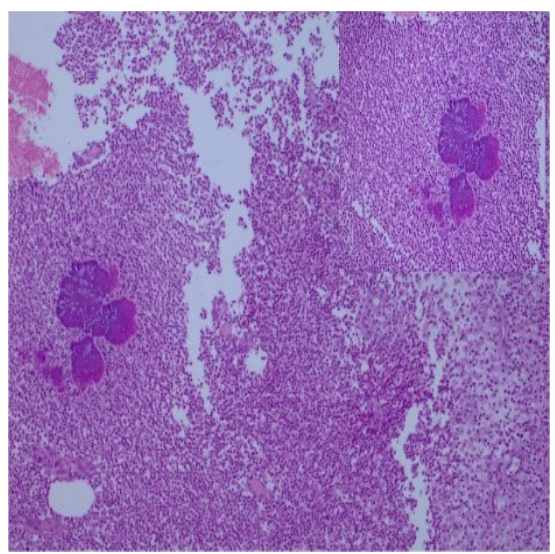

Fig.3: Histopathological image

\section{CASE REPORT}

A 54-year-old female patient presented to our outpatient clinic with 3 different months of diffuse intermittent colic-style abdominal pain, constipation, and involuntary weight loss of approximately $10 \%$ of total body weight during the 3 months. There was no history of any known disease or operation. The patient was postmenopausal and had no history of IUD use. The patient had signs of obstruction and on physical examination, the abdomen was distended with palpable stiff mass lesion filling the right lower quadrant and extending to the umbilicus. Moreover, there was no guarding or rebound. The laboratory parameters of infection were in the normal range at the time of admission (WBC: 8300 CRP: $3.2 \mathrm{mg} / \mathrm{L}$ ) other than mild anemia (Hb: 10.1 g/dl Hct: 32.5\%). A subsequent contrast-enhanced computed tomography (CT) revealed a redundant sigmoid colon, which was extended to the midline level of the umblicus with a mass lesion invading its entire length. The mass was also extended to the mesocolon and mesenteric root and there were enlarged lymph nodes (figure 1).

On colonoscopic examination, stenosis was observed at the level of sigmoid colon, which did not allow the passage of the colonoscope. No intraluminal lesion was seen. Laparotomy was planned for the patient. During the exploration with a midline incision, a giant conglomerate mass lesion of approximately $18 \mathrm{~cm}$ in diameter, located near the rectosigmoid region, and containing the cecum and small intestine mesentery was seen (figure 2). The appendix was also observed to be adherent to the mass. Anterior resection with anastomosis and an appendectomy was performed. No other pathological findings were observed and the operation was completed. There were no problems in the postoperative follow-up visit. Oral intake was started, bowel movements were present, and on the 5th day, the patient was discharged without any problems. The patient's 1-month postoperative follow-up was uneventful.

The pathological examination of the specimen revealed especially neutrophil, plasma cell, eosinophil, and lymphocyte infiltration, multinuclear giant cells, scarcely suppurative granuloma, microabscess areas, and an actinomycosis-compatible bacteria in the subserosal areas and fat tissue (figure 3). The findings were thought to be consistent with diverticulitis and diverticulum perforation. There were 35 reactive lymph nodes in the specimen. No features were observed in the appendix.

\section{DISCUSSION}

Abdominal actinomycosis is a rare condition characterized by chronic, purulent, granulomatous inflammation caused by Actinomyces Israeli. It usually occurs between the ages of 20-60 years and is three times more common in men than women. The annual incidence is reported as $1 / 300.000$. 5,6 Actinomyces have low virulence. For the occurrence of the disease, a predisposing factor that causes mucosal damage or immunosuppression such as 
infection, malignancy, trauma, or IUD is needed. ${ }^{7}$

In our case, the patient was considered to have no predisposing factor before the diagnosis but on pathological examination, sigmoid diverticulitis with no clinical signs was found after the operation. It is very difficult to diagnose the disease preoperatively and only $10 \%$ of all patients can be diagnosed before the operation. ${ }^{8}$ Most cases like ours present with an abdominal mass. It is not possible to differentiate actinomycosis from other inflammatory and neoplastic pathologies by the laboratory, endoscopic, or radiological examinations. ${ }^{9}$ CT is the most commonly used diagnostic method for finding the location and content of the lesion. It can be observed intra- or extraluminal, and it is difficult to distinguish it from a malignant mass when it is accompanied by an intense desmoplastic reaction. Especially in patients with chronic, mild clinical course, infiltrative lesion irregularity should be suspected of actinomycosis. ${ }^{7,9}$ Using the aspiration fluid taken by imaging, direct microscopic examination, or culture of actinomyces can be done. However, because of the bacterial diversity in the intestinal flora, they are not helpful methods. ${ }^{10}$ As the disease usually develops extraluminally, a colonoscopic examination is not helpful in the diagnosis. However, endoscopic examination is useful to rule out colitis and tumor pathologies. Sometimes fibrosis can be seen in the bowel wall due to nodular lesions and chronic inflammation. ${ }^{11}$ Because of this, many cases are diagnosed as malignant tumors in the preoperative period. Diagnosis is based on microscopic examination of the specimen to find sulfur granules or gram-positive filament organisms, or isolation of the actinomyces species from the culture done from the specimen. ${ }^{12}$ If the diagnosis can be made without surgery and the case is not complicated, the preferred treatment is antibiotherapy. High-dose penicillin is the standard treatment, and cephalosporins can be used less frequently. Surgical resection is usually performed before antibiotherapy because of the difficulties in the diagnosis and it is seen that $90 \%$ of cases have a complete recovery after antimicrobial therapy. ${ }^{13}$ Long-term antibiotic treatment is required because of the recurrence, but there is no consensus on which drug to choose and how long the treatment would last. Usually, penicillin or its derivatives are used, after at least 1 week of intravenous treatment, oral antibiotherapy can be started and the treatment is continued for a minimum of 2 months. ${ }^{14} \mathrm{We}$ also started treatment in a similar way for our patient and planned to follow a long time therapy as recommended. Abdominal actinomycosis is a very rare disease, but it should be considered in the differential diagnoses of patients with intra-abdominal mass. Actinomyces infection is likely to be seen as an opportunistic infection, especially in cases of diverticulitis and the like. In our case, diverticulitis and diverticulitis perforation were the initiating processes. As it is hard to diagnose preoperatively, tomographic screening and needle aspiration biopsy may be diagnostic.

When the diagnosis can be made, first of all, surgical treatment should be reserved for stubborn and complicated cases that do not benefit from medical treatment. Surgical treatment can be considered as the first choice in the presence of large necrotic tissue, especially if malignancy is not excluded, as in our patient.

In conclusion, more studies are needed to more accurately diagnose abdominal actinomycosis. We presented this case to increase the awareness about this disease, which is possible to be treated non-surgically and should be suspected in the differential diagnoses.

\section{ETHICAL APPROVAL}

There is nothing to be declared.

\section{CONFLICT OF INTEREST}

The authors declare no conflict of interest related to this work.

\section{REFERENCES}

1. Berardi RS. Abdominal actinomycosis. Surg Gynecol Obstet 1979;149:257-66.

2. Israel J. Neue Beobachtungen auf dem Gebiete der Mykosendes Menschen. Arch Path Anat Physiol Klin Med 1978;74:15-53.

3. Belmont MJ, Behar PM, Wax MK. Atypical presentations of actinomycosis. Head Neck 1999;21:264-8. doi: 10.1002/(sici)10970347(199905)21:3<264::aid-hed12>3.0.co;2-y.

4. Yi F, Prasad S, Sharkey F, Kahlenberg M. Actinomycotic infection of the abdominal wall mimicking a malignant neoplasm. Surg Infect 2008 ;9:85-9. doi: 10.1089/sur.2006.055.

5. Wong VK, Turmezei TD, Weston VC. Actinomycosis BMJ 2011;343:d6099-90. doi: 10.1136/bmj.d6099.

6. A cevedo F, Baudrand R, Letelier LM, Gaeteb P. Acti- 
nomycosis: a great pretender. Case reports of unusual presentations and a review of the literature. Int J Infect Dis 2008;12:358-62. doi: 10.1016/j.ijid.2007.10.006.

7. Karateke F, Ozyazıcı S, Menekşe E, Daş K, Ozdoğan M. Unusual presentations of actinomycosis; anterior abdominal wall and appendix: report of three cases. Balkan Med J 2013;30:315-7. doi: 10.5152/balkanmedj.2012.377.

8. Garcia-Garcia A, Ramirez-Duran N, Sandoval-Trujillo H, Romero-Figueroa MDS. Pelvic Actinomycosis. Can J Infect Dis Med Microbiol 2017;2017: 9428650. doi: 10.1155/2017/9428650.

9. McFarlane ME, Coard KC. Actinomycosis of the colon with invasion of the abdominal wall: An uncommon presentation of a colonic tumour. Int J Surg Case Rep 2010;1:9-11. doi: 10.1016/j.ijscr.2010.07.002.

10. M. Saitoh, N. Kanoh, E. Wada. A case of ileocecal actinomycosis with areview of 37 cases reported in Japan. Jpn Soc Coloproctol 1990;43:613-20. doi: 10.3862/jcoloproctology.43.613.

11. Kim JC, Ahn BY, Kim HC, Yu CS, Kang GH, Ha HK, et al. Efficiency of combined colonoscopy and computed tomography for diagnosis of colonic actinomycosis: a retrospective evaluation of eight consecutive patients. Int J Colorectal Dis 2000;15:236-42. doi: 10.1007/ s003840000232.

12. Russo TA . Agents of actinomycosis. In: Mandel GL, Bennett JE, Dolin R, eds. Principles and practice of infectious diseases. 7th edn: Elsevier Churchill Livingstone, 2010:3209-19.

13. Brook I. Actinomycosis: diagnosis and management. South Med J 2008;101:1019-23. doi: 10.1097/ SMJ.0b013e3181864c1f.

14. Wagenlehner FM, Mohren B, Naber KG, Männl HF. Abdominal actinomycosis. Clin Microbiol Infect 2003;9:881-5. doi: 10.1046/j.1469-0691.2003.00653.x 